\title{
To forge a solid immune recognition
}

\author{
Yan Shi \\ Department of Basic Medical Sciences, School of Medicine, Tsinghua University, Beijing 100084, China \\ Department of Microbiology, Immunology and Infectious Diseases; Snyder Institute, University of Calgary, T2N 1N4, Alberta, \\ Canada \\ $\triangle$ Correspondence: yanshi@biomed.tsinghua.edu.cn
}

Phagocytosis and innate immune responses to solid structures are topics of interest and debate. Alum, monosodium urate, calcium pyrophosphate dehydrate, silica and by extension all solid entities draw varying degrees of attention from phagocytes, such as antigen presenting cells. For some, innocuous soluble metabolites turn into fierce irritants upon crystallization, pointing to divergent signaling mechanisms of a given substance in its soluble and solid states. Over the years, many mechanisms have been proposed, including phagocytic receptors, toll like receptors, and NACHT-LRRs (NLRs), as well as several other protein structure mediated recognition of the solids. Is there a more general mechanism for sensing solids? In this perspective, I present an alternative view on the topic that membrane lipids can engage solid surfaces, and the binding intensity leads to cellular activation. I argue from the stands of evolution and biological necessity, as well as the progression of our understanding of cellular membranes and phagocytosis. The effort is to invite debate of the topic from a less familiar yet equally thrilling viewing angle.

\section{CURRENT IMMUNOLOGICAL VIEW OF SOLID PARTICLE PHAGOCYTOSIS}

Solid structures either introduced exogenously or as a result of metabolite precipitation in vivo, invoke varying degrees of response. At one extreme such as in solid adjuvants, alum and monosodium urate (MSU) crystals, or particle based drug delivery, their intense interactions with tissues are mostly desirable. On the other hand, such responses are detrimental in artificial implants and silicosis. The mechanism of immune recognition of solid structures has not been well conceptualized. It lacks the theoretical frameworks that adorn other events such as antigen recognition ( $T$ cell receptor/major histocompatibility complex or TCR/MHC), T cell development (positive and negative selection) and innate immunity (pathogen-associated molecular patterns/toll-like receptors or PAMPs/TLRs). At the point of initial contact between a solid particle and a phagocyte, several mechanisms have been proposed to explain the signaling events. The most prevalent is the host factor coating of these solids. Antibody Fc chains, sugar moieties and complement fragments present on the surfaces of solids can trigger their corresponding receptors on the phagocytes and lead to particle uptake. This is called opsonization which likely covers a large portion of the observed phagocytic phenomenon. Difficulty comes where some cells that lack those receptors are also phagocytic, and most solid particles are engulfed in defined buffers or media without the participation of soluble components. Post industrial materials without evolutionarily conceivable receptors trigger phagocytosis just as efficiently in vivo and in vitro. On the other hand, phagocytosis is a common biological activity that predates the mammalian immune system. It presents a question: what had happened before those opsonizing mechanisms became available? The search for a more generic mechanism for detecting solid structures has been a lonely inquiry in a protein centric research field. At the present time, all mammalian phagocytosis is presumed to be receptor driven events. I would like to argue that the receptor based solid structure phagocytosis is a leaky proposal if placed under the backdrop of evolution.

I will not discuss cell surface receptors in phagocytosis as our focus lies aside. However, intracellular sensing deserves some clarification. Recent development in immunology reveals the role of inflammasome in solid particle mediated inflammation (Martinon et al., 2009). Research in this direction has gained great attention. It has been loosely suggested 
that inflammasomes, such as NLRP3/ASC/Caspase-1 complex that controls IL-1 $\beta$ production, can "sense" solid structures. There is no consensus about what the "sense" is. Intracellular sensing is not satisfying for two more reasons. Prior to the activation of inflammation or the activation of inflammasome, a large set of events have already taken place, including phagocytosis signaling, cytoskeleton rearrangement, membrane exchange between cell surface and intracellular vesicles, and full fledged activation of antigen presenting cells (APCs) (Ng et al., 2008; Flach et al., 2011). These events are not accounted for by the inflammasome activation. What is the sensor for these events? The question remains as to what makes solid particles "seen" by phagocytes at the first place. Even if inflammasomes were the sensor of solid structures, how did they meet each other? As a side note, several recent papers reported that NLRP3 inflammasome is not essential for the adjuvant effect of solid alum particles (Franchi and Núñez, 2008; McKee et al., 2009; Flach et al., 2011; Guarda et al., 2011; Kuroda et al., 2011), implicating a different sensing mechanism.

This opinion piece tries to combine some experimental observations with theoretical extrapolations to suggest one possibility: in some cases, membrane lipid binding may trigger cellular activation. One of the consequences is phagocytosis and possibly immune activation.

\section{THROUGH THE LENS OF EVOLUTION}

In 1882, Elie Metchnikoff introduced thorns from a tangerine tree into the larvae of starfish and saw them surrounded by mobile cells after a few hours (Karnovsky, 1981). It occurred to him that those "amoeboid wandering cells" could be used as a form of defense against "noxious intruders," an idea proven worthy of the Nobel years later. He extended his work into leukocytes and observed their ability to engulf microbes, a phenomenon he called "phagocytosis." He also called large phagocytes "macrophages" and smaller ones "microphages" (the latter has turned out to be polymorphonuclear cells). Cellular immunity was then founded as a research discipline.

Phagocytosis predates immunologically defined phagocytic receptors and our immune system by at least one billion years. Eukaryogenesis (circa 2 billion years ago) itself has been thought to be the result of phagocytosis as cellular organelles such as mitochondria likely came from engulfment of prokaryotic life forms (Yutin et al., 2009). Multicellularity dates as far back as one billion years. This was the earliest time that we can reasonably attribute all modern receptor based phagocytosis to, although it was more likely a feature of the later animal kingdom (Fig. 1). From a cell biology view point, the epitome of phagocytosis is the remodeling of cytoskeleton; an elemental yet complex process that much resembles chemotaxis (Yutin et al., 2009). At the center of these events are the changes in cell shape and of intracellular structure rearrangements, an "advanced" life style only afforded by the "newly" emerged cytoskeleton during eukaryogenesis. As early as amoebozoa and before the age of animals, plants and fungi, phagocytosis was the means of energy and nutrient uptake, and likely not a protein receptor mediated event (Yutin et al., 2009).

In contrast to this basic function of biology, phagocytosis in animals has evolved into a scavenging and host defense mechanism. The classification of phagocytic receptors, FcR (immunoglobulin superfamily), mannose receptors (C-type lectins, carbohydrate binding protein), complement receptors (a collection of different protein family members), etc. are phylogenically scattered (Underhill and Ozinsky, 2002). It is reasonable to assume that these receptors were developed, along with the host immunity, as a set of "niche" functions rather than a bona fide driving force of the genesis of phagocytosis. It is therefore not surprising that phagocytic receptors have not evolved as a coherent stream; rather they are secondary to other activities, such as antibody binding and complement fixation and became the phenomenon of opsonization.

If phagocytosis is an ancient cellular function, we are then faced with a question: how did they interact with solids at the beginning? Have advanced immune systems abandoned this primordial art? or are we immunologists looking the other way in favor of receptor based mechanism? We can break down the discussion into several segments.

\section{THROUGH THE LENS OF LIPID BIOLOGY}

Let's ask questions in detail: without receptor, how does a cell know what to do when in contact with a solid structure? What is the nature of contact without a receptor/ligand interaction? How does it transduce signals across the plasma membrane? Except for chemotaxis (Chung et al., 2001), we actually know little about this fundamental cellular event that has been with us since the eukaryogenesis. Assuming cytoskeletal changes may come as a result of exoplasma membrane contact with a foreign object; there are a few generic ways that we may hypothesize how these two events are linked, i.e. electrostatic charges, hydrophobic interactions and generic chemical interactions such as hydrogen bonds and Van der Waals force between a solid structure and the outer leaflet of the plasma membrane. Essentially, these mechanisms lead to the same thing: binding to the cell surface. It is reasonable to assume that in the absence of a receptor-like presence, binding to the cell membrane is the first step for phagocytic activation. What happens afterwards is not well worked out. It shall prove helpful to review some basic features of the biological bilayer membrane.

To set the premise, it should be pointed out that eukaryogenesis was also marked by the first appearance of cholesterol in the membrane (although fatty acids with multi ring side chains are present in archaea). Sphingolipids are present throughout biology. The arrival of cholesterol and to- 


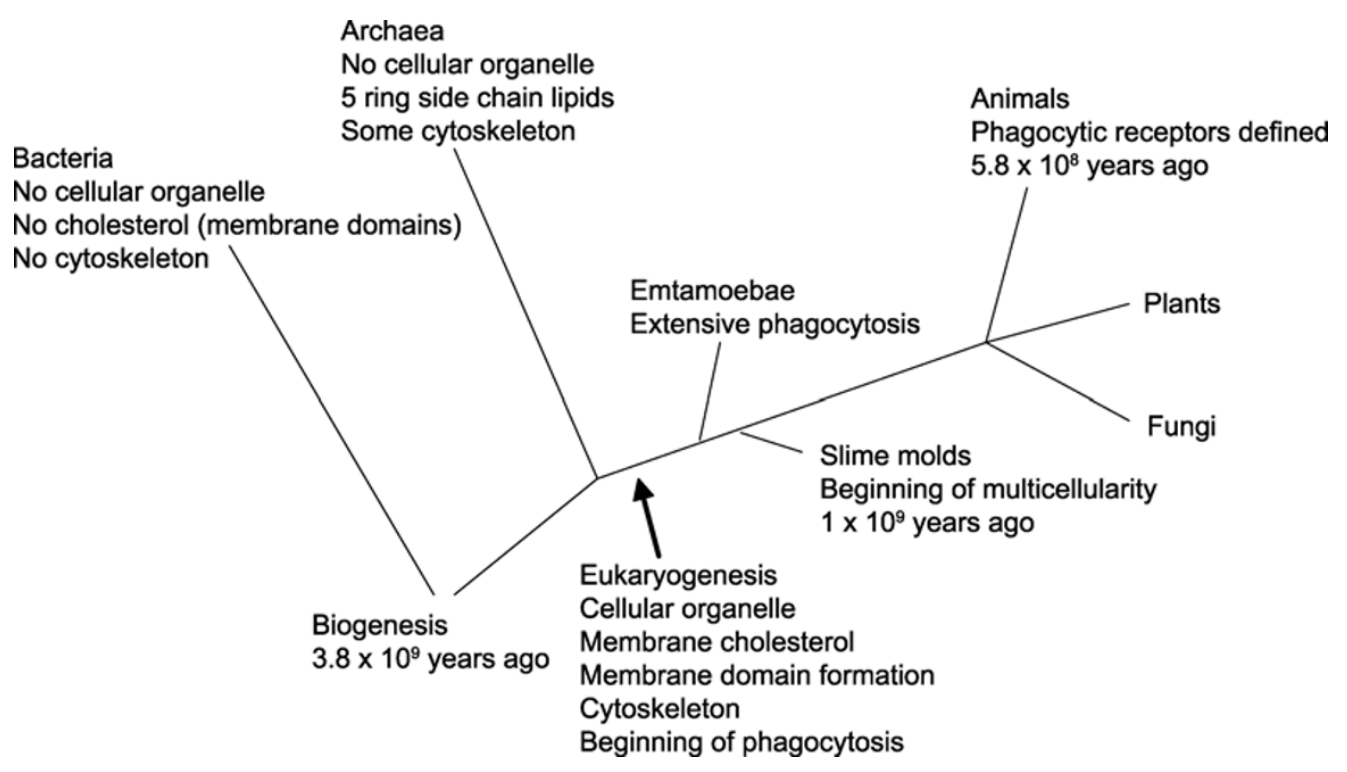

Figure 1. Phylogenesis of phagocytosis. An abstract depiction of phagocytosis and eukaryogenic evolution. The time lines are approximate. The phenomenon of membrane affinity triggered signaling (MATS) likely took place right after eukaryogenesis.

gether with sphingolipids and other saturated lipids provided the building materials for lipid sorted domains (lipids rafts), and dictates critical behavior of the eukaryotic membrane. The main feature and perhaps the evolutionary incentive of its existence is cholesterol's ability to form stable contact with saturated aliphatic chains (Simons and Vaz, 2004). With only anemic understanding of it, I would venture to say this is the essence of membrane domain formation, a staple of eukaryotic life style.

Singer and Nicolson's model of mosaic bilayer lipids depicts a hydrophobic core made of aliphatic chains of fatty acids and hydrophilic outer membranes occupied by head groups (Singer and Nicolson, 1972). The original model proposed the insertion of transmembrane proteins in the lipid membrane. But it appeared inadequate almost as soon as it was proposed, as the plasma membrane is dynamic and "non-ideal" (Pike, 2009). The concept of lipid domains first appeared in the work of Kai Simons and others in the 70s and 80 s, mainly with the study of epithelial cells (Simons and van Meer, 1988; Levental et al., 2010). Simons and others realized that lipid vesicles coming off the Golgi were different, and the differences could be used to explain the divergent lipid compositions between apical and basolateral cell membranes. It was also thought that the lipid composition at each surface could be maintained against diffusion by exchanging vesicles among them. Inconspicuously, Simons suggested that newly synthesized proteins could be sorted by these lipid domains for targeting purposes, and the presence of proteins in the lipid bilayer created curvatures that reciprocally determined the feature of lipid vesicles. This last point suggested the interplay between lipids and surface proteins and has been proven correct, and becomes most relevant in our discussion. Readers are referred to a review for details (Pike, 2009).

There are two features maybe of relevance to lipid initiated signaling. (1) The bilayer membrane is attached to the cytoskeletal structures. (2) Some proteins are preferentially sorted into the lipid domains. In either case, a resting cell will look dramatically different from an activated cell from the perspective of membrane lipid arrangement. Prior to activation, the lipid domains are extremely small (a few dozen nanometers) and short in half life (seconds). Each domain will unlikely contain more than one protein molecule judging from their respective sizes, let alone two identical ones. Such a minuscule scale and scarcity of between signaling motifs is the basic means to keep a cell in its resting state. Ligand binding is to change this state and triggers large lipid/protein domain formation.

The above description of lipid membrane is utilitarian, but the hope is to establish the chronological order of cellular activation-lipid mediated cellular functions had long been there before any modern phagocytic and immune receptors made it to the scene.

\section{AMOEBA'S TABLE MANNERS AND SIGNALING EQUIVALENT PLATFORM (SEP): A THOUGHT EXPERIMENT}

For life forms as simple as amoeba, taking external substances inside must have been a top order of work because it was the way of feeding. Nonetheless, the elemental features of a eukaryotic bilayer membrane were present then. In addition, essential components of phagocytic activities were pre- 
sent as well. Wortmannin and cytochalasin D block amoeba uptake of particles, suggesting that signaling events akin to $\mathrm{PI} 3 \mathrm{~K}$ and actin polymerization happened in the ancient form of phagocytosis (Akya et al., 2009). Eukaryotic chemotaxis is a direct result of actin activities, which may explain the movement of early phagocytes toward particulate targets and topological formation of pseudopods engulfing the targets (physical constrain of a solid structure may force the formation of pods as chemotaxis continues). It is unlikely to explain all cases of phagocytosis as crystalline structures represent a large part of the earth (minerals and soil). Modern day amoeba phagocytose latex beads well (Avery et al., 1995). Therefore having a "solid diet" is in their genes. The remaining questions are: (1) Is there any evidence that solids can bind to lipids, (2) Do lipids sort as a consequence of binding? (3) Can protein receptors signal following lipid domain sorting? We have evidence for each of these points.

A long time ago, Neil Mendal believed that the crystal surface interaction was a pure surface attraction between plasma membrane and crystal lattice. In fact, he attempted to categorize the intensity of crystal mediated responses by their surface molecular densities and packing patterns (Mandel, 1976). Hydrogen bonds between crystal surface and phospholipids were suggested as the main mechanism (Mandel and Mandel, 1976). In one assay, he addressed the issue of spatial matching between atomic repeats of crystal surface and the density of lipid heads on the plasma membrane (this is where protein based crystal recognition also fails as the protein receptor densities are logs lower than the functional repeats on the crystal surface) (Mandel, 1994). It was suggested that cholesterol is essential for binding. In the study of kidney stones, it was also clear that renal epithelial cell surface lipids were essential for the binding (Bigelow et al., 1996, 1997; Koka et al., 2000). Working on this hypothesis, our lab measured the binding between alum/MSU crystals and individual cholesterol and sphingomyelin molecules and quantitatively addressed the intensity of these forces $(\mathrm{Ng}$ et al., 2008). Lipid affinity for crystal surfaces is therefore well supported.

The second point, lipids sorting as a consequence of binding, has been proven beyond any doubt and is in itself of discipline of research. Roux et al. reported that random mixtures of lipids on a model membrane can be sorted into different phases under the vacuum force (Roux et al., 2005). Driving kinesin along a microtubule will also generate enough forces to sort lipids (Sorre et al., 2009). Therefore, if sufficient binding exists between lipid moieties and a crystal surface, lipid sorting is an expected outcome. In fact there are many ways membrane lipids can be sorted as a consequence of either a binding force or an introduction of curvature (McMahon and Gallop, 2005). Our work with MSU and alum suggests the surfaces of crystals attached to plasma membrane can rearrange the membrane lipids as well and this rearrangement does signal ( $\mathrm{Ng}$ et al., 2008; Flach et al., 2011).
The last point, receptor signaling following lipid domain sorting, is at this stage an extrapolation. The concept behind is a divergence from canonical ligand triggered receptor conformation change or dimerization. Akihiro Kusumi proposes that protein receptor signaling, rather than a self contained event, is a means to achieve lipid domain aggregation (Kusumi et al., 2004; Kusumi and Suzuki, 2005). Essentially, this in a way predicts a 'synapse' like entity for all membrane signaling events. Here, there is no difference between a ligand/receptor pair induced higher order lipid domain or one produced by a membrane curvature or any other biophysical means. The central purpose is to bring together enough sorted lipids and their associated protein receptors, and signaling ensues. He calls the means to achieve this final platform "stabilizers." The recently developed concept of critical behavior of plasma membrane strongly supports his proposal. It has been proposed that real cell membranes under physiological conditions are very close to the state of "critical point" with reference to the lipid aggregation and diffusion (Gordon et al., 2008). A critical point, as a physical concept, refers to a substance or a mixture, being at a state between two settled phases. Minimal external energy is required to drive towards either direction. For the real plasma membrane, this state refers to the large scale lipid demixing (domain formation). While lipid driven signaling molecule clustering has been a concept in existence for long time, the revelation of mammalian lipid membrane being near its critical point explains why a receptor ligation by its ligand, minute on the scale of energy, is sufficient to drive large scale lipid rearrangement and protein aggregation (Harder and Engelhardt, 2004; Nielsen et al., 2004; John and Bär, 2005; Magal et al., 2009). It is likely that the affinity between a solid surface and membrane lipids is another way to achieve the same outcome.

We therefore assume lipid sorting can take place in the absence of receptors. As such we may expect to see an aggregation of signaling molecules that are utilized in cytoskeleton movement. This might be the ancient form of phagocytosis, which may result in a platform suggested in Fig. 2. The question is: is this shape and composition really different from the end product of receptor ligand interaction (Fig. 2; left and right)? Using the example of APC phagocytosis: lipid domain aggregation, ITAM containing protein receptors, Syk and PI3K, and the end result of phagocytosis are essentially indistinguishable. I would like to propose that these two structures are signaling-wise equivalent (signaling equivalent platform or SEP). This may explain why solid structures trigger activation similar to receptor ligand interactions. If simple surface binding mediated phagocytosis predated the receptor based counterpart, did protein receptors invent this complex chain of events or did phagocytic receptors evolve to take the advantage of the pre-existing mechanism? It appears more likely that phagocytic receptors evolved to use this ancient mechanism and developed the phenomenon of phagocytosis 

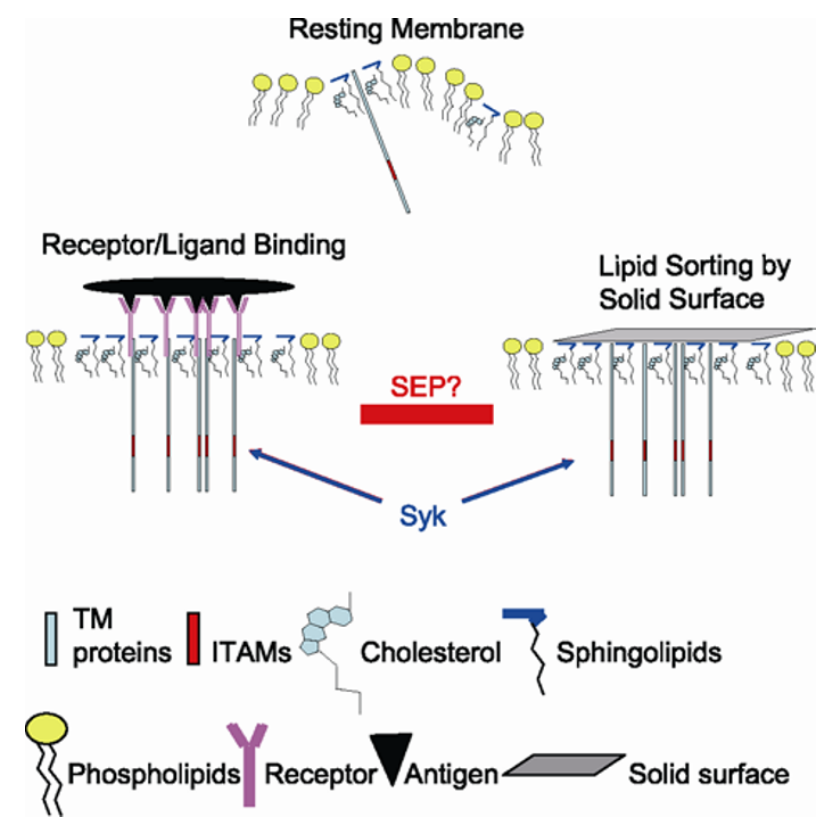

Figure 2. Signaling Equivalent Platform. A hypothetical mechanism of lipid sorting by topological reasoning: free state of lipid rafts are enriched with proteins of signaling potential, such as ITAM containing receptors. These domains are admixed with other non-raft lipids and are randomly dispersed. Packing cholesterol and sphingolipids continuously may produce a flat planar surface. However it does not happen as it is energetically unfavourable. Upon binding of a receptor/ligand, or being stabilized by an approaching solid surface, phase separation will cluster these two lipids and drive out other species. Thus, the lipid rafts and their associated proteins are sorted to high density for signaling. To a phagocyte, the left and the right are equivalent for its activation. ITAM, immunoreceptor tyrosine-based activation motif.

as defined by immunologists. However, if modern phagocytes are atavistically activated by simple lipid binding, will they heed this call? Is this the basis of phagocytic response to solid structures by modern APCs?

\section{ON SOLID GROUND?}

Membrane lipid alteration has biological consequences. In fields such as anesthesiology (Ueda and Kamaya, 1984; Urban et al., 2006) and mechanosensation (Martinac, 2004), lipid change as the onset of signaling is de novo point of investigation. Anesthetics work by altering membrane lipid fluidity and therefore protein functions in the membrane. Changes in tension of bilayer membrane alter the shape of ion channels permitting electrolyte influx. This is the basis of our mechanosensing (how we feel shape and force).

In spite of these parallels, the proposal of lipid mediated particle sensing should be expanded beyond crystal recogni- tion with great caution. Looking closely at home, several thorny points appear, and the proposal is still on a shaky ground because of them: (1) If lipid binding is the starting point and assuming most cells have similar plasma membrane composition, why are only some activated? (2) How does phagocytosis of non-solid structures (such as cellular debris) work? (3) What happens when solids are covered by proteins, lipids and other natural and synthetic materials? The answer to all of them is we do not know. It is possible that with the development of multicellular life forms, only cells that may come in contact with external objects retain the phagocytosis ability, while others would have suppressed it. Regarding questions 2 and 3 , if one uses tight atomic repeats of crystal surface as the match for tightly adjacent lipid heads, one must remember that this will work against a similar explanation for phagocytosis of covered crystals as the surface lattice is likely hidden. Do more dispersed ionic interactions amongst lipids, proteins and carbohydrates work to the same effect as crystals binding? Does opsonization cover the rest? There are legitimate possibilities. However, while this piece argues that the lipid contact as the potential starting point of phagocytosis is a worthy proposition from an evolutionary angle, its applicability in modern day phagocytosis as a whole is likely to be common but not ubiquitous. It is likely that while lipid based sensing mechanism could explain the origin of phagocytosis and may still work at times, opsonization based mechanisms have become the main player in vivo and can explain the majority of phagocytic events observed in the lab.

\section{REMARKS}

The lipid based explanation for solid structure sensing could be wrong or merely an epiphenomenon in phagocytosis. One day, other mechanisms may come to disprove this proposal, which shall be welcome. This opinion piece is an effort to provide alternative mechanisms as receptor based ones, as discussed here, cannot be the last word.

A theory's reach wanes when its power of explaining becomes strained and unnatural, as I believe it is in terms of receptor based recognition of solid structures. The long list of potential mechanisms can mean extreme redundancy. It can also mean that a simple explanation is not yet available. The role of lipid based immune recognition is not a conceptual leap of faith, but an acknowledgement that lipid based biological events studied in our neighboring disciplines have relevance in our field. For the lack of proper categorization of this class of events, Wajahat Mehal and I tentatively call it membrane affinity triggered signaling or MATS. Although research tools are limited and workers few, our willingness to probe this new direction will overcome obstacles. As a personal inclination, treating membrane lipid behaviors as a feature of immunology is not only prudent, but may prove to be a common denominator beneath many immunologic functions. 


\section{ACKNOWLEDGEMENTS}

The author is funded by the National Institutes of Health, USA (No. R21AI089963 and R01Al098995), Canadian Institutes of Health Research (No. MOP-119295) and Natural Sciences and Engineering Research Council of Canada (No. RGPIN/355350), and thanks Dr. Robin Yates, Dr. Yang Yang, and Fay Munro for their comments and assistance.

\section{REFERENCES}

Akya, A., Pointon, A., and Thomas, C. (2009). Mechanism involved in phagocytosis and killing of Listeria monocytogenes by Acanthamoeba polyphaga. Parasitol Res 105, 1375-1383.

Avery, S.V., Harwood, J.L., and Lloyd, D. (1995). Quantification and Characterization of Phagocytosis in the Soil Amoeba Acanthamoeba castellanii by Flow Cytometry. Appl Environ Microbiol 61, 1124-1132.

Bigelow, M.W., Wiessner, J.H., Kleinman, J.G., and Mandel, N.S. (1996). Calcium oxalate-crystal membrane interactions: dependence on membrane lipid composition. J Urol 155, 1094-1098.

Bigelow, M.W., Wiessner, J.H., Kleinman, J.G., and Mandel, N.S. (1997). The dependence on membrane fluidity of calcium oxalate crystal attachment to IMCD membranes. Calcif Tissue Int 60, 375-379.

Chung, C.Y., Funamoto, S., and Firtel, R.A. (2001). Signaling pathways controlling cell polarity and chemotaxis. Trends Biochem Sci 26, 557-566.

Flach, T.L., Ng, G., Hari, A., Desrosiers, M.D., Zhang, P., Ward, S.M., Seamone, M.E., Vilaysane, A., Mucsi, A.D., Fong, Y., et al. (2011). Alum interaction with dendritic cell membrane lipids is essential for its adjuvanticity. Nat Med 17, 479-487.

Franchi, L., and Núñez, G. (2008). The NIrp3 inflammasome is critical for aluminium hydroxide-mediated IL-1beta secretion but dispensable for adjuvant activity. Eur J Immunol 38, 2085-2089.

Gordon, V.D., Deserno, M., Andrew, C.M.J., Egelhaaf, S.U., and Poon, W.C.K. (2008). Adhesion promotes phase separation in mixed-lipid membranes. EPL 84, 48003.

Guarda, G., Braun, M., Staehli, F., Tardivel, A., Mattmann, C., Förster, I., Farlik, M., Decker, T., Du Pasquier, R.A., Romero, P., et al. (2011). Type I interferon inhibits interleukin-1 production and inflammasome activation. Immunity 34, 213-223.

Harder, T., and Engelhardt, K.R. (2004). Membrane domains in lymphocytes - from lipid rafts to protein scaffolds. Traffic 5, 265-275.

John, K., and Bär, M. (2005). Travelling lipid domains in a dynamic model for protein-induced pattern formation in biomembranes. Phys Biol 2, 123-132.

Karnovsky, M.L. (1981). Metchnikoff in Messina: a century of studies on phagocytosis. N Engl J Med 304, 1178-1180.

Koka, R.M., Huang, E., and Lieske, J.C. (2000). Adhesion of uric acid crystals to the surface of renal epithelial cells. Am J Physiol Renal Physiol 278, F989-F998.
Kuroda, E., Ishii, K.J., Uematsu, S., Ohata, K., Coban, C., Akira, S., Aritake, K., Urade, Y., and Morimoto, Y. (2011). Silica crystals and aluminum salts regulate the production of prostaglandin in macrophages via NALP3 inflammasome-independent mechanisms. Immunity 34, 514-526.

Kusumi, A., Koyama-Honda, I., and Suzuki, K. (2004). Molecular dynamics and interactions for creation of stimulation-induced stabilized rafts from small unstable steady-state rafts. Traffic 5, 213-230.

Kusumi, A., and Suzuki, K. (2005). Toward understanding the dynamics of membrane-raft-based molecular interactions. Biochim Biophys Acta 1746, 234-251.

Levental, I., Grzybek, M., and Simons, K. (2010). Greasing their way: lipid modifications determine protein association with membrane rafts. Biochemistry 49, 6305-6316.

Magal, L.G., Yaffe, Y., Shepshelovich, J., Aranda, J.F., de Marco, Mdel.C., Gaus, K., Alonso, M.A., and Hirschberg, K. (2009). Clustering and lateral concentration of raft lipids by the MAL protein. Mol Biol Cell 20, 3751-3762.

Mandel, N. (1994). Crystal-membrane interaction in kidney stone disease. J Am Soc Nephrol 5, S37-S45.

Mandel, N.S. (1976). The structural basis of crystal-induced membranolysis. Arthritis Rheum 19, 439-445.

Mandel, N.S., and Mandel, G.S. (1976). Monosodium urate monohydrate, the gout culprit. J Am Chem Soc 98, 2319-2323.

Martinac, B. (2004). Mechanosensitive ion channels: molecules of mechanotransduction. J Cell Sci 117, 2449-2460.

Martinon, F., Mayor, A., and Tschopp, J. (2009). The inflammasomes: guardians of the body. Annu Rev Immunol 27, 229-265.

McKee, A.S., Munks, M.W., MacLeod, M.K., Fleenor, C.J., Van Rooijen, N., Kappler, J.W., and Marrack, P. (2009). Alum induces innate immune responses through macrophage and mast cell sensors, but these sensors are not required for alum to act as an adjuvant for specific immunity. J Immunol 183, 4403-4414.

McMahon, H.T., and Gallop, J.L. (2005). Membrane curvature and mechanisms of dynamic cell membrane remodelling. Nature 438, 590-596.

Ng, G., Sharma, K., Ward, S.M., Desrosiers, M.D., Stephens, L.A., Schoel, W.M., Li, T., Lowell, C.A., Ling, C.C., Amrein, M.W., et al. (2008). Receptor-independent, direct membrane binding leads to cell-surface lipid sorting and Syk kinase activation in dendritic cells. Immunity 29, 807-818.

Nielsen, S.O., Lopez, C.F., Ivanov, I., Moore, P.B., Shelley, J.C., and Klein, M.L. (2004). Transmembrane peptide-induced lipid sorting and mechanism of Lalpha-to-inverted phase transition using coarse-grain molecular dynamics. Biophys J 87, 2107-2115.

Pike, L.J. (2009). The challenge of lipid rafts. J Lipid Res 50, S323-S328.

Roux, A., Cuvelier, D., Nassoy, P., Prost, J., Bassereau, P., and Goud, B. (2005). Role of curvature and phase transition in lipid sorting and fission of membrane tubules. EMBO J 24, 1537-1545.

Simons, K., and van Meer, G. (1988). Lipid sorting in epithelial cells. Biochemistry 27, 6197-6202.

Simons, K., and Vaz, W.L. (2004). Model systems, lipid rafts, and cell 
membranes. Annu Rev Biophys Biomol Struct 33, 269-295.

Singer, S.J., and Nicolson, G.L. (1972). The fluid mosaic model of the structure of cell membranes. Science 175, 720-731.

Sorre, B., Callan-Jones, A., Manneville, J.B., Nassoy, P., Joanny, J.F., Prost, J., Goud, B., and Bassereau, P. (2009). Curvature-driven lipid sorting needs proximity to a demixing point and is aided by proteins. Proc Natl Acad Sci U S A 106, 5622-5626.

Ueda, I., and Kamaya, H. (1984). Molecular mechanisms of anes- thesia. Anesth Analg 63, 929-945.

Underhill, D.M., and Ozinsky, A. (2002). Phagocytosis of microbes: complexity in action. Annu Rev Immunol 20, 825-852.

Urban, B.W., Bleckwenn, M., and Barann, M. (2006). Interactions of anesthetics with their targets: non-specific, specific or both? Pharmacol Ther 111, 729-770.

Yutin, N., Wolf, M.Y., Wolf, Y.I., and Koonin, E.V. (2009). The origins of phagocytosis and eukaryogenesis. Biol Direct 4, 9. 